PROCEEDINGS OF THE

AMERICAN MATHEMATICAL SOCIETY

Volume 124, Number 3, March 1996

\title{
ON A CONVOLUTION INEQUALITY OF SAITOH
}

\author{
MICHAEL CWIKEL AND RONALD KERMAN
}

(Communicated by Andrew M. Bruckner)

Abstract. Let $F_{1}, F_{2}, \ldots, F_{j}, \ldots$ be in the class $L_{\mathrm{loc}}\left(\mathbb{R}_{+}\right)$of locally integrable functions on $\mathbb{R}_{+}=(0, \infty)$. Define the convolution product $\prod_{j=1}^{m} * F_{j}$ inductively by $\left[\prod_{j=1}^{2} * F_{j}\right](x)=\left(F_{1} * F_{2}\right)(x)=\int_{0}^{x} F_{1}(y) F_{2}(x-y) d y$ and $\prod_{j=1}^{m} * F_{j}=\left[\prod_{j=1}^{m-1} * F_{j}\right] * F_{m}$ for $m>2$. The inequality

$$
\int_{0}^{\infty} x^{-(m-1)(p-1)}\left|\left[\prod_{j=1}^{m} * F_{j}\right](x)\right|^{p} d x \leq[(m-1) !]^{1-p} \prod_{j=1}^{m} \int_{0}^{\infty}\left|F_{j}(y)\right|^{p} d y
$$

is obtained for each $p, 1<p<\infty$. Further, the constant $[(m-1) !]^{1-p}$ is shown to be the best possible, and the nonzero extremal functions are determined.

\section{INTRODUCTION}

Let $F_{1}, F_{2}, \ldots, F_{j}, \ldots$ be in the class $L_{\mathrm{loc}}\left(\mathbb{R}_{+}\right)$of complex-valued locally integrable functions on $\mathbb{R}_{+}=(0, \infty)$ (i.e., they are integrable on $(0, r)$ for each $\left.r>0\right)$. Define the convolution product $\prod_{j=1}^{m} * F_{j}$ by $\left[\prod_{j=1}^{1} * F_{j}\right]=F_{1},\left[\prod_{j=1}^{2} * F_{j}\right](x)=$ $\left(F_{1} * F_{2}\right)(x)=\int_{0}^{x} F_{1}(y) F_{2}(x-y) d y$ and inductively for $m>2$ by $\prod_{j=1}^{m} * F_{j}=$ $\left[\prod_{j=1}^{m-1} * F_{j}\right] * F_{m}$. It is easy to check that each of the functions $\prod_{j=1}^{m} * F_{j}$ must also be in $L_{\mathrm{loc}}\left(\mathbb{R}_{+}\right)$.

Our result here is

Theorem. Fix $p, 1<p<\infty$. Then, for each positive integer $m$,

$$
\int_{0}^{\infty} x^{-(m-1)(p-1)}\left|\left[\prod_{j=1}^{m} * F_{j}\right](x)\right|^{p} d x \leq[(m-1) !]^{1-p} \prod_{j=1}^{m} \int_{0}^{\infty}\left|F_{j}(x)\right|^{p} d y .
$$

The constant $[(m-1) !]^{1-p}$ is best possible. Moreover the (nonzero ) extremal functions are of the form $F_{j}(y)=C_{j} e^{-c y}$ a.e., where $C_{j}$ are constants and $\operatorname{Re} c>0$, $j=1, \ldots, m$.

The above result is known in the case $p=2$ and $m$ even, where it was proved by Saitoh [5] using Aronszajn's theory of reproducing kernels.

Received by the editors November 24, 1993 and, in revised form, July 17, 1994.

1991 Mathematics Subject Classification. Primary 26D90.

Key words and phrases. Convolution, Titchmarsh theorem.

The first author's research was supported by the Fund for Promotion of Research at the Technion.

The second author's research was supported by NSERC grant A4021.

(C)1996 American Mathematical Society 
Our proof of the above theorem uses Hölder's inequality, Titchmarsh's convolution theorem, and the well-known functional equation for exponential functions.

\section{THE PROOF OF THE THEOREM}

For $n \geq 1$ we have

$$
\begin{aligned}
& \int_{0}^{\infty} x^{-n(p-1)}\left|\left[\prod_{j=1}^{n+1} * F_{j}\right](x)\right|^{p} d x \\
& \quad=\int_{0}^{\infty} x^{-n(p-1)} d x\left|\int_{0}^{x} y^{(n-1) / p^{\prime}} y^{(1-n) / p^{\prime}}\left[\prod_{j=1}^{n} * F_{j}\right](y) F_{n+1}(x-y) d y\right|^{p}
\end{aligned}
$$

where $p^{\prime}=\frac{p}{p-1}$. Applying Hölder's inequality to the inner integral we obtain that the above expression is dominated by

$$
\int_{0}^{\infty} x^{-n(p-1)} \frac{x^{n(p-1)}}{n^{p-1}} d x \int_{0}^{x} y^{-(n-1)(p-1)}\left|\left[\prod_{j=1}^{n} * F_{j}\right](y)\right|^{p}\left|F_{n+1}(x-y)\right|^{p} d y .
$$

Applying Fubini's theorem to this integral we see that we have shown

$$
\begin{aligned}
& \int_{0}^{\infty} x^{-n(p-1)}\left|\left[\prod_{j=1}^{n+1} * F_{j}\right](x)\right|^{p} d x \\
& \quad \leq n^{1-p} \int_{0}^{\infty} y^{-(n-1)(p-1)}\left|\left[\prod_{j=1}^{n} * F_{j}\right](y)\right|^{p} d y \int_{0}^{\infty}\left|F_{n+1}(t)\right|^{p} d t .
\end{aligned}
$$

We can now obtain (1.1) by induction. The case $m=1$ is trivial. The case $m=2$ is simply (2.1) with $n=1$. If (1.1) holds for $m=n$, then (2.1) yields that

$$
\begin{aligned}
& \int_{0}^{\infty} x^{-n(p-1)}\left|\left[\prod_{j=1}^{n+1} * F_{j}\right](x)\right|^{p} d x \\
& \quad \leq n^{1-p}[(n-1) !]^{1-p} \prod_{j=1}^{n} \int_{0}^{\infty}\left|F_{j}(y)\right|^{p} d y \int_{0}^{\infty}\left|F_{n+1}(t)\right|^{p} d t \\
& \quad=(n !)^{1-p} \prod_{j=1}^{n+1} \int_{0}^{\infty}\left|F_{j}(y)\right|^{p} d y
\end{aligned}
$$

completing the proof of (1.1).

Now we determine under what conditions equality can hold in (1.1), apart from the obvious and trivial cases where $m=1$ or $m>1$ and one or more of the functions $F_{j}$ vanish a.e. Equality in (1.1) implies that equality holds in (2.1) for each positive integer $n$ with $n \leq m-1$. This happens only if equality holds in Hölder's inequality, i.e., only if for a.e. $x>0$ there exists a number $k(x) \in \mathbb{C}$ such that

$$
y^{(1-n) / p^{\prime}}\left[\prod_{j=1}^{n} * F_{j}\right](y) F_{n+1}(x-y)=k(x) y^{(n-1) / p}
$$


for a.e. $y \in(0, x)$. It is convenient to rewrite this in the form:

$$
\text { For a.e. } x \in \mathbb{R}_{+} f(y) g(x-y)=k(x) \text { for a.e. } y \in(0, x) \text {, }
$$

where $g=F_{n+1}$ and $f(y)=y^{1-n}\left[\prod_{j=1}^{n} * F_{j}\right](y)$.

Our next step is to prove that $k: \mathbb{R}_{+} \rightarrow \mathbb{C}$ is a measurable function. This is not quite as obvious as it might seem at first. Observe that (2.3) is not automatically the same as saying $f(x) g(x-y)=k(x)$ for a.e. $x$ in some interval, for various fixed values of $y$. (See Remark 2.7 below for further discussion of this matter.) Our proof will use an auxiliary function of the form

$$
h(x, y):=\frac{U(x)}{x} \cdot \chi_{\{(x, y) \mid 0<y<x\}}(x, y) \cdot V(\operatorname{Re}\{f(y) g(x-y)\}) .
$$

We need $U$ to be integrable and strictly positive with $\int_{0}^{\infty} U(x) d x=1$, and $V$ : $\mathbb{R} \rightarrow \mathbb{R}$ must be continuous, strictly monotone, and bounded (e.g. take $U(x)=e^{-x}$ and $V(t)=\arctan t)$. Clearly $h$ is an integrable function on $\mathbb{R}_{+} \times \mathbb{R}_{+}$. So, by Fubini's theorem, the function $H(x):=\int_{0}^{\infty} h(x, y) d y$ must be an integrable and thus measurable function of $x$ on $\mathbb{R}_{+}$. But, by (2.3), we have $H(x)=U(x) V(\operatorname{Re} k(x))$ for a.e. $x \in \mathbb{R}_{+}$. Consequently $\operatorname{Re} k(x)=V^{-1}\left(\frac{H(x)}{U(x)}\right)$ must be measurable. Similarly $\operatorname{Im} k(x)$ is also measurable, and therefore so is $k$.

Now we can deduce that the nonnegative function

$$
\varphi(x, y)=|f(y) g(x-y)-k(x)| \cdot \chi_{\{(x, y) \mid 0<y<x\}}(x, y)
$$

must also be measurable on $\mathbb{R}_{+} \times \mathbb{R}_{+}$. So we can apply Tonelli's theorem (i.e., Fubini's theorem for nonnegative but not necessarily integrable functions) to $\varphi$. Using $(2.3)$ we obtain first that $f(y) g(x-y)=k(x)$ for a.e. $(x, y)$ in $\{(x, y): 0<$ $y<x\}$. Equivalently we have that

$$
f(\alpha) g(\beta)=k(\alpha+\beta)
$$

holds for a.e. $(\alpha, \beta)$ in the set $\mathbb{R}_{+} \times \mathbb{R}_{+}$. We also obtain that for a.e. $y \in \mathbb{R}_{+}$, $f(y) g(x-y)=k(x)$ for a.e. $x \in(y, \infty)$. This implies that $k$ must be locally integrable on $\mathbb{R}_{+}$since $g$ is.

We have excluded the case where $g$ vanishes a.e. and we can also assume that $f$ is nonzero on some subset of positive measure of $\mathbb{R}_{+}$since otherwise, by Titchmarsh's theorem [6] (see also [2], [3]), at least one of the functions $F_{1}, F_{2}, \ldots, F_{n}$ would have to vanish a.e. Thus, by the Lebesgue differentiation theorem, there exist intervals $\left[\alpha_{0}, \alpha_{1}\right]$ and $\left[\beta_{0}, \beta_{1}\right]$ in $\mathbb{R}_{+}$such that $\int_{\alpha_{0}}^{\alpha_{1}} f(t) d t$ and $\int_{\beta_{0}}^{\beta_{1}} g(t) d t$ are both nonzero. We deduce that $f(\alpha)$ coincides for a.e. $\alpha \in \mathbb{R}_{+}$with the continuous function

$$
f_{1}(\alpha):=\int_{\beta_{0}}^{\beta_{1}} k(\alpha+t) d t / \int_{\beta_{0}}^{\beta_{1}} g(t) d t
$$

and analogously $g(\beta)$ coincides for a.e. $\beta \in \mathbb{R}_{+}$with the continuous function $g_{1}(\beta):=\int_{\alpha_{0}}^{\alpha_{1}} k(t+\beta) d t / \int_{\alpha_{0}}^{\alpha_{1}} f(t) d t$. Now for every positive $t$ and $r$ and for every positive $\alpha$ and $\beta$ such that $t=\alpha+\beta$ we have

$$
\frac{1}{r^{2}} \int_{0}^{r} \int_{0}^{r} k(t+x+y) d x d y=\frac{1}{r^{2}} \int_{0}^{r} \int_{0}^{r} f_{1}(\alpha+x) g_{1}(\beta+y) d x d y .
$$

We deduce that the limit $k_{1}(t):=\lim _{r \rightarrow 0} \frac{1}{r^{2}} \int_{0}^{r} \int_{0}^{r} k(t+x+y) d x d y$ exists for every 
$t>0$ and satisfies

$$
k_{1}(\alpha+\beta)=f_{1}(\alpha) g_{1}(\beta)
$$

for all positive $\alpha$ and $\beta$. Clearly $k_{1}(t)=f_{1}\left(\frac{1}{2}\right) g_{1}\left(\frac{t}{2}\right)$ and so is a continuous function on $\mathbb{R}_{+}$.

We claim that all of the functions $f_{1}(t), g_{1}(t)$, and $k_{1}(t)$ are nonzero for every $t>0$. Suppose not; then $k_{1}(\delta)=0$ for some $\delta>0$, and either $f_{1}\left(\frac{\delta}{2}\right)$ or $g_{1}\left(\frac{\delta}{2}\right)$ must also vanish. Then, for every $\gamma>\frac{\delta}{2}$ we have $k_{1}(\gamma)=f_{1}\left(\gamma-\frac{\delta}{2}\right) g_{1}\left(\frac{\delta}{2}\right)=$ $f_{1}\left(\frac{\delta}{2}\right) g_{1}\left(\gamma-\frac{\delta}{2}\right)=0$ and thus $k_{1}$ vanishes on the interval $\left[\frac{\delta}{2}, \infty\right)$. By reiterating this argument sufficiently many times we see that $k_{1}(t)=0$ for each $t>0$. As explained above, each of the functions $f$ and $g$ is nonzero on some set of positive measure. This implies that the same is true for $f_{1}$ and $g_{1}$. But this contradicts (2.5) for some values of $\alpha$ and $\beta$ and so proves our claim.

It now follows, again using (2.5), that the limits $f_{1}(0+)$ and $g_{1}(0+)$ both exist and are nonzero. Hence the function $H(x):=f_{1}(x) / f_{1}(0+)=g_{1}(x) / g_{1}(0+)$ satisfies $H(x-y) H(y)=k_{1}(x) / f_{1}(0+) g_{1}(0+)=H(x)$ for all $0<y<x$. This implies that $f_{1}(x)=f_{1}(0+) e^{-c x}$ and $g_{1}(x)=g_{1}(0+) e^{-c x}$, where $c$ is a constant satisfying $\operatorname{Re} c>0$. See [1], pp. 35-36.

In particular, setting $n=1$, the above argument shows that

$$
F_{j}(x)=C_{j} e^{-c x} \quad \text { a.e. }
$$

for $j=1,2$. Now, if (2.6) holds for $j=1,2, \ldots, n$, then clearly

$$
\left[\prod_{j=1}^{n} * F_{j}\right](y)=\text { const. } y^{n-1} e^{-c y} .
$$

But also, again by the preceding argument, $\left[\prod_{j=1}^{n} * F_{j}\right](y)=$ const. $y^{n-1} e^{-c^{\prime} y}$ and $F_{n+1}(y)=$ const. $e^{-c^{\prime} y}$ for some constant $c^{\prime}$. It follows that $c^{\prime}=c$, which shows that (2.6) also holds for $j=n+1$, and so, by induction, for all $j=1,2, \ldots, m$.

Remark 2.7. The proof of measurability of $k$ given above may seem somewhat indirect. Let us indicate some difficulties which are encountered if we attempt to give a more direct proof. Let $F(x, y)=f(y) g(x-y) \chi_{T}(x, y)$ and $K(x, y)=$ $k(x) \chi_{T}(x, y)$, where $T=\{(x, y): 0<y<x\}$, and let $N=\left\{(x, y) \in \mathbb{R}_{+} \times \mathbb{R}_{+}\right.$: $F(x, y) \neq K(x, y)\}$. For each $x>0$ define the $x$-section $N_{x}=\{y>0:(x, y) \in N\}$, and for each $y>0$ define the $y$-section $N^{y}=\{x>0:(x, y) \in N\}$. To show that $k$ is measurable it would suffice to show that $N^{y}$ has zero measure for each $y$ in some sequence tending to zero. We know from (2.3) that $N_{x}$ has zero measure for a.e. $x>0$. If we knew that $N$ were a measurable subset of $\mathbb{R}_{+} \times \mathbb{R}_{+}$, then we could immediately apply Tonelli's theorem to obtain that $N$ has zero planar measure and consequently $N^{y}$ has zero measure for a.e. $y$. But to show that $N$ is measurable we need to know what we are trying to prove, namely that $k$ is measurable. As a further indication of the possible difficulties here, we mention the example due to Sierpinski (see [4], p. 167) of a nonmeasurable subset $Q$ of $[0,1] \times[0,1]$ all of whose $x$ - and $y$-sections are measurable subsets of $[0,1]$. In fact, $Q_{x}$ has measure 1 and $Q^{y}$ has measure 0. So if we define $P=\{(x, y):(y, x) \in Q\}$ and then $\widetilde{N}=\bigcup_{m \geq 0, n \geq 0} P+(m, n)$ we obtain that $\widetilde{N}^{y}$ has infinite measure for each $y>0$ even though $\widetilde{N}_{x}$ has measure 0 for each $x>0$. 


\section{REFERENCES}

1. A. Aczel, Lectures on functional equations and their applications, Academic Press, New York and London, 1966.

2. J. L. Lions, Supports de produits de composition, C. R. Acad. Sci. Paris 232 (1951), 16221624. MR 13:231a

3. J. G. Mikusinski, A new proof of Titchmarsh's theorem on convolution, Studia Math. 13 (1953), 56-58. MR 15:407g

4. W. Rudin, Real and complex analysis, 3rd ed. McGraw-Hill, New York, 1986. MR 88k:00002

5. S. Saitoh, A fundamental inequality in the convolution of $L_{2}$ functions on the half line, Proc. Amer. Math. Soc. 91 (1984), 285-286. MR 85j:30010

6. E. C. Titchmarsh, The zeros of certain integral functions, Proc. London Math. Soc. 25 (1926), 283-302.

Department of Mathematics, Technion-Israel Institute of Technology, Haifa, 32000, ISRAEL

E-mail address: mcwikel@techunix.technion.ac.il

Department of Mathematics, Brock University, St. Catharines, Ontario, Canada L2S 3A1

E-mail address: rkerman@spartan.ac.brocku.ca 